\title{
The Impact of Higher Order Sectorisation on the Performance of Millimetre Wave 5G Network
}

\author{
Naser Al-Falahy and Omar Y. K. Alani \\ School of Computing, Science \& Engineering \\ University of Salford \\ Manchester, United Kingdom \\ N.F.A.AL-Falahy@edu.salford.ac.uk,O.Y.K.Alani@salford.ac.uk
}

\begin{abstract}
The fifth Generation (5G) mobile network will provide services with extreme data rate and latency demands compared to current cellular network, and provide massive capacity and connectivity to multitude of devices with diverse requirements and applications. Therefore, it is important to utilise all network resources to provide the $5 \mathrm{G}$ vision. In this paper, performance evaluations and impact of higher order horizontal sectorisation on next generation 5G mobile access is presented. The study has been focused on busy urban areas in high carrier frequency. Millimetre wave band has precious wide unexploited bandwidth that can be harnessed for mobile communication. The results for these scenarios show that higher-order horizontal sectorisation in millimetre wave based smallcell deployment can significantly increase the network capacity to meet the future requirement of 5G network, and provide high data rate and connectivity to huge number of devices. Moreover, beamforming can highly increase the data rate by efficiently increase signal power and suppress interference from unwanted directions.
\end{abstract}

Index Terms -5G network, millimetre wave, network densification, higher order sectorisation, beamforming.

\section{INTRODUCTION}

Wireless broadband traffic growth has introduced major impact on future mobile network architectures, and hence, it requires more demands. These demands include: (i) High traffic volume (massive capacity): increases on the order of several magnitudes. The future requirement is a 1000x increase in data traffic for 2020 and beyond. (ii) Increased indoor and smallcells/hotspot traffic: will be the majority of mobile traffic volume. Today, about 70 percent of mobile traffic happens indoors; in the future, indoor data traffic as well as hotspot area may exceed this figure. (iii) Higher number of connected devices (massive connectivity): will be created from huge connected subscribers and from machine-to-machine (M2M) applications, as all devices that benefit from internet traffic need to become connected. (iv) Energy consumption: $5 \mathrm{G}$ need to be a green network to reduce carbon footprint [1], [2].

Mobile communication has transformed from only voice service into a complex inter-connected environment with multi services, built on a system that support multitude of applications and provide high speed access to massive number of subscribers [3], [4].

High order horizontal sectorisation in low carrier frequency has been studied previously in [5] and [6] for old mobile generation as a way to increase frequency reuse and improve network capacity. In addition, vertical sectorisation (see fig.1) has its benefits in maximizing network capacity [7] and provide better coverage solution for high rise buildings [8].

This paper is organized as follows. Section II presents work motivation and challenges. Section III introduces our network model including directional antenna and millimetre wave band. Section IV explains simulation parameter, network elements, and results. And the impact of beamforming has been discussed in section V. Finally, conclusions are drawn in Section VI.

\section{Motivation AND CHALlenges}

\section{A. Motivation}

Due to the adoption of highly directional antennas at millimetre wave bands, higher order sectorisation (HOS) will be a promising solution to increase network resources and system coverage at these bands. Higher order sectorisation will ensure enough resources to cope with the massive increase in mobile data traffic and to provide a network with massive capacity and connectivity.

\section{B. Challenges}

The adoption of millimetre wave band as a potential spectrum for $5 \mathrm{G}$ will require radical change to the network architecture due to the very different propagation condition and hardware constraint. Furthermore, the huge traffic expected to be generated due to the higher sectorised basestation operating in millimetre wave band should be backhauled to the core network. The current wired and wireless traditional backhaul links are not a viable solution due to their limited capacity and design requirements. 
Optical fibre links on the other hand, can provide the capacity when connecting the smallcells dense deployment. However, bringing a fibre connection to the smallcell nodes is laborious and expensive, as the trend of this deployment is with high density. Thus, the provision of a highly efficient and flexible wireless backhaul link to replace the current optical fibre links is a promising alternative [9].

\section{NETWORK MODEL}

Sectorisation is a widely used technique for increasing the capacity in wireless cellular networks by spatial reuse of radio resources [10] with typical configuration being a three sectored solution [5].

Unlike conventional network planning in the legacy $<3 \mathrm{GHz}$ spectrum, HOS seems a promising key enabler to improve network capacity and data throughput, as millimetre wave tends to use highly directional antennas with high gain to compensate for the high pathloss at these bands. In our model, different scenarios (sectorisation order) were simulated. The focus of this work is on meeting the high data throughput demands required from $5 \mathrm{G}$ network. The performance of the following scenarios has been compared:

- Three horizontal sectors - Four horizontal sectors

- Six horizontal sectors - Eight horizontal sectors

- Ten horizontal sectors

\section{A. Directional Antennas}

In order to compensate for a large pathloss and penetration loss of mm-wave signals, we use directional antennas with a high antenna gain.

A linear array antenna has been used by [11] for simpler analysis and a rectangular planar array antenna has been used by [12] as a more practical directional antenna. Furthermore, horn antenna can be used as a high gain solution in millimetre wave as described in [13]. We use a horn antenna as a directional beamforming pattern obtained through XFdtd, a full wave 3D simulation tool [14] as shown in fig. 2 .

\section{B. Millimetre wave smallcells}

Since the overwhelming majority of communication systems are already operating in the microwave band (MW) below $3 \mathrm{GHz}$, due to its favourable propagation characteristics. This makes MW band too scarce. Therefore, $5 \mathrm{G}$ system is anticipated to use mm-wave bands, particularly (20-90) $\mathrm{GHz}$, due to the availability of wide chunk of unused bandwidth. This step is revolutionary because mm-wave frequency band has very different propagation condition, atmospheric absorption and hardware constraint. Such challenges, however, could be compensated using beamforming and larger antenna array.
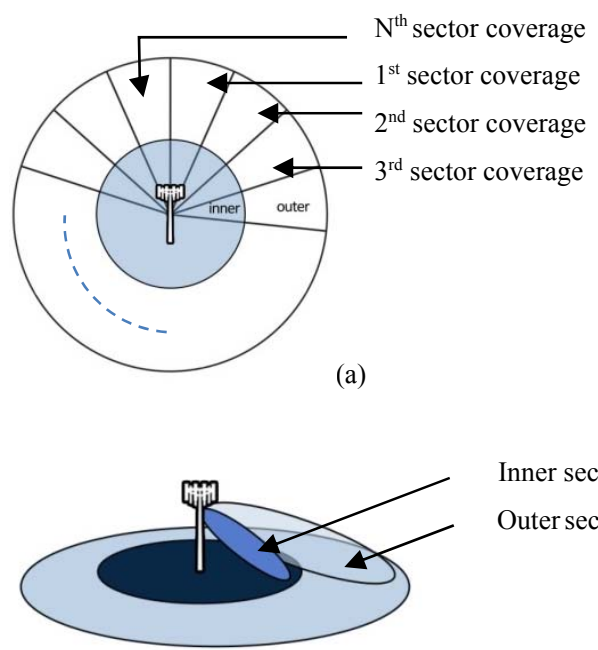

Inner sector coverage Outer sector coverage

(b)

Fig.1. High order Sectorisation, (a) Horizontal Sectorisation, and (b) Vertical Sectorisation

It is widely accepted that mm-wave need to be used with very limited cell radius $<200 \mathrm{~m}$, to minimize high pathloss at this band (by bringing the access point closer to the user). Fortunately, this action fits well with the dense deployment of smallcell which will be the trend of next generation mobile system [15]. As per our model, smallcell with high order sectorisation is considered as shown in fig.1 (a); the coverage of smallcell area is divided equally by the HOS.

\section{Simulation AND ReSUlts}

Simulations are carried out by Wireless InSite tool [16], a ray-tracing analyser and high-fidelity EM solvers for radio network planning, a $130 \mathrm{~m}$ inter site distance (ISD) has been selected based on recent contribution [15], [17], [18] with random UE distribution and network share the available resources among users according to the received SNR for each user equipment (UE) and the load factor. A $300 \times 300 \mathrm{~m}^{2}$ area is considered, and due to high pathloss and penetration loss at millimetre wave band, $1^{\text {st }}$ non-line-of-site (NLOS) is considered to be $15 \mathrm{~dB}$ weaker than line-of-site (LOS), $2^{\text {nd }}$ NLOS is further reduced by another $15 \mathrm{~dB}$, other reflections are excluded from analysis due to their very weak signals. Details of parameters are listed in table I.
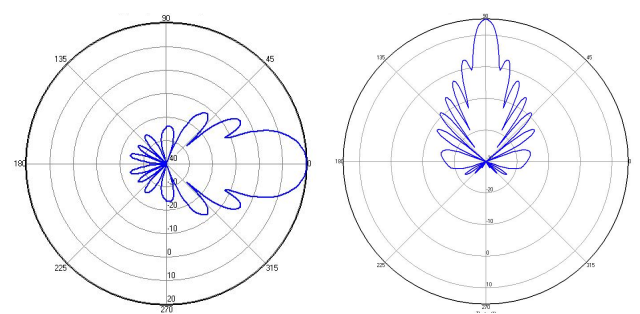

Fig.2, Beamforming patterns, left is horizontal and right is vertical. 
The data throughput of a user in a cellular network is upper-bounded by Shannon channel capacity theorem [19]:

$$
\mathrm{C}_{\mathrm{ch}}=\mathrm{m}(\mathrm{B} / \mathrm{n}) \log _{2}(1+\mathrm{SINR})
$$

where $\mathrm{m}$ is the number of spatial streams between a base station and user, $\mathrm{B}$ is the signal bandwidth, $\mathrm{n}$ (loading factor) is the number of users served by a base station, and SINR is the received signal power, to interference plus noise ratio, respectively. In this study, the scenario was conducted in a busy urban area as it is the first choice of $5 \mathrm{G}$ deployment, assuming (600) users to be outdoor, distributed in in the streets of the city as shown in fig.3.

The number of spatial link (m) is assumed to be 1 and the load factor (n) is variable from scenario to scenario and region to region, however, when load factor is small, user within this area experience data speed in excess of multi Gbps (see fig.4). Therefore, sectorisation with wide bandwidth $(1 \mathrm{GHz})$ at millimetre wave band is a promising solution to the massive increase in mobile data traffic.

TABLE I. NETWORK MODEL PARAMETERS.

\begin{tabular}{|l|l|}
\hline Model parameter & Value \\
\hline Tx Power & $30 \mathrm{dBm}$ \\
\hline Tx antenna gain & $15 \mathrm{~dB}$ \\
\hline Tx Pattern & As per fig.2 \\
\hline Antenna type & SISO, 2x2 MIMO \\
\hline Rx antenna gain & $2 \mathrm{~dB}$ \\
\hline Rx pattern & $\begin{array}{l}\text { i- Omni } \\
\text { ii- }- \text { Beamforming }\end{array}$ \\
\hline Carrier frequency & $28 \mathrm{GHz}$ \\
\hline Bandwidth & $500 \mathrm{MHz}$ \\
\hline Tx Antenna height & $8 \mathrm{~m}$ \\
\hline Rx antenna height & $1.5 \mathrm{~m}$ \\
\hline No. of Rx & $600 \mathrm{randomly} \mathrm{distributed}$ \\
\hline Area of steady & $(300 \mathrm{x} 300) \mathrm{m}^{2}$ \\
\hline Noise Figure & $8 \mathrm{~dB}$ \\
\hline Noise Density & $-174 \mathrm{dBm} / \mathrm{Hz}$ \\
\hline Rx Thermal noise & $-74 \mathrm{dBm}$ \\
\hline Sectorisation & 3 to 10 \\
\hline Scheduler & Proportional Fair \\
\hline
\end{tabular}

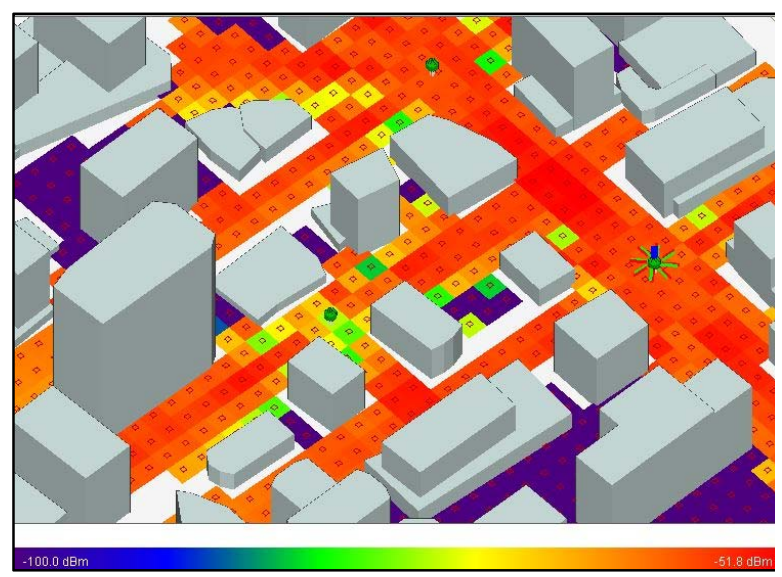

Fig.3, Network model, urban area with 600 user distribution

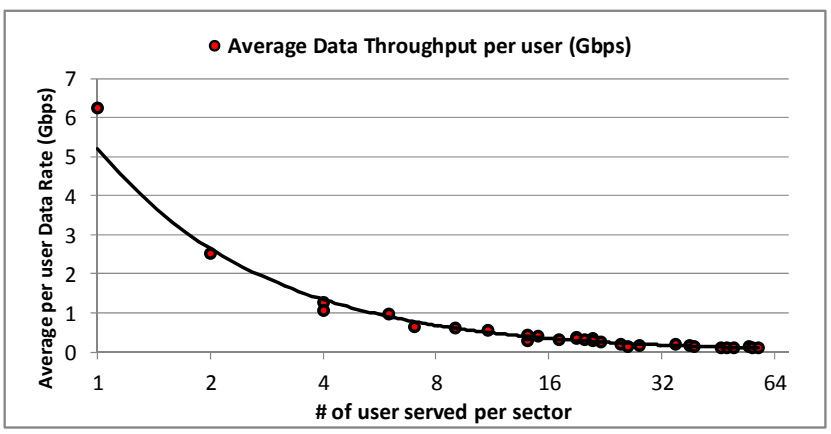

Fig.4, Average data throughput per user vs. loading factor

The number of user served by sectorised site is increasing with the increase of sectorisation order, which is known as the sectorisation gain, the sectorisation has another advantage in distributing user around nearby cells and sectors, which eventually minimize the load factor and increase the data throughput as a result, fig. 4 demonstrate this case. However, this relation does not hold linearly, as the sectorisation order increase, the interference from nearby sectors and sites increase consequently, therefore, at the $10^{\text {th }}$ order of sectorisation; the number of users receiving (fair) SINR has decreased (SINR values are shown in fig.5), which affect the coverage probability in LOS and NLOS regions, as shown in fig.6.

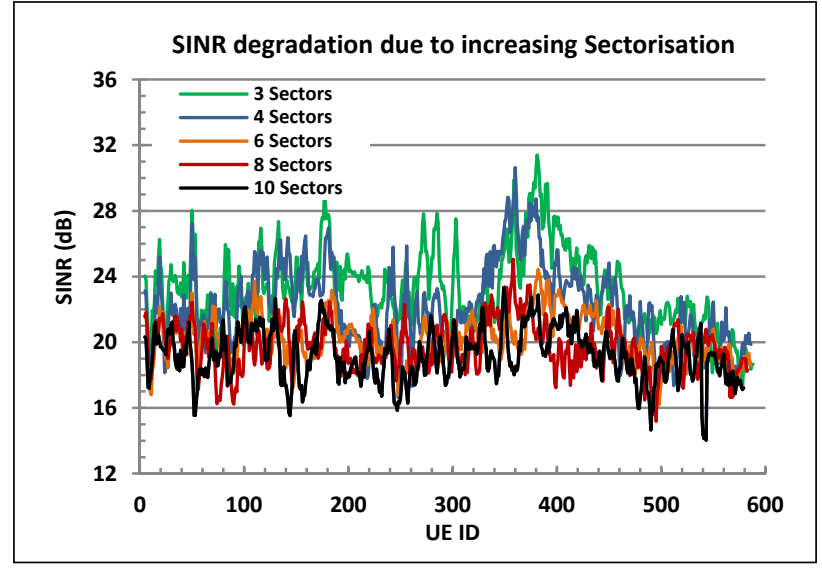

Fig.5, Average data throughput per user vs. loading factor

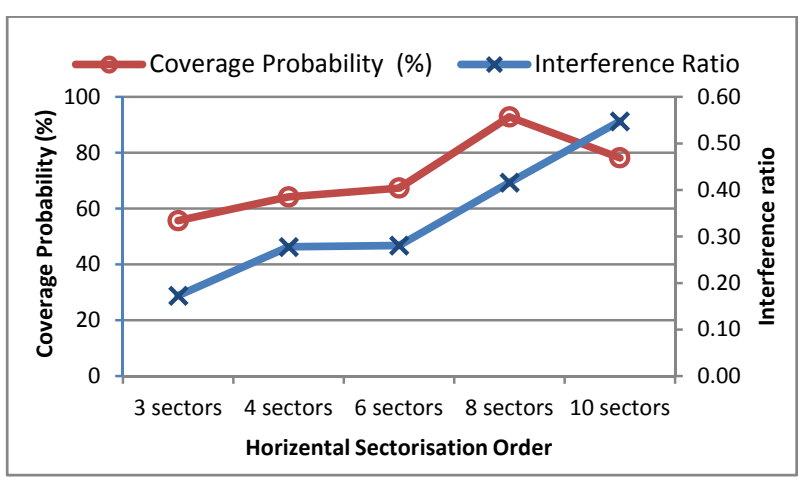

Fig.6, Base station coverage probability vs. interference in different site topologies 
Therefore, an eight order sectorisation is favourable choice to maintain coverage probability while increasing the data speed in certain geographical area. If, for specific case, a more data rate is required in a hot spot due to their extreme demands, a vertical sectorisation is recommended which will boost the 8 sector site data speed, through orthogonal polarized antennas that provide orthogonal beams to user (horizontal polarization (HP) and vertical polarization VP)), so that the same system bandwidth is utilised for both sectors as shown in Fig.1 b, and defined in equation (2). Here, the resources are doubled in the polarized beams:

$$
\mathrm{B}_{\mathrm{T}}=\mathrm{B}_{\text {inn (HP) }}=\mathrm{B}_{\text {out (VP) }}
$$

where $\mathrm{B}_{\mathrm{T}}$ is the total bandwidth and $\mathrm{B}_{\mathrm{inn}(\mathrm{HP})}, \mathrm{B}_{\mathrm{out}(\mathrm{VP})}$ are the bandwidths of inner and outer vertical sectors, respectively.

Increasing the probability of having LOS exist is highly recommended in millimetre wave communications; high order sectorisation has increased the coverage probability with LOS transmission. In table II, the LOS coverage probabilities as well as the overall coverage probability for different site topologies are shown.

It's worth mentioning here, that new site (smallcell) deployment to improve LOS coverage outperform sectorisation results as the operator has full control on site location, planning and optimization to provide the best link.

Therefore, dense deployment of smallcells in millimetre wave band is a key enabler for $5 \mathrm{G}$ massive connectivity to provide access channel to a massive number of machines and devices to enable the internet-of-Things (IoT). However, the resulted interference should be managed with interference cancellation techniques, here, we will introduce beamforming as a way to supress unwanted signal, as clarified in the next section.

\section{BEAMFORMING}

Beamforming $(\mathrm{BF})$ is the concentration of power in certain direction, which will increase wanted signal power and suppress other signals (interference) efficiently. Beamforming in millimetre wave is key enabling technologies of next generation $5 \mathrm{G}$ system, as they can significantly improve signal strength, which can lead to higher cell throughput and better cell edge performance compared to $4 \mathrm{G}$ network.

Beamforming can be used in base station (TxBF) as well as UE (RxBF), as future mm-wave handset could be developed with higher number of antennas that can beamform and steer their reception and transmission with higher gain and efficiency. One of the advantages of HOS recently discussed is that it reduces the losses due to beam steering, for instance, in Tx beamforming; base station will steer its beams to their parented subscribers for maximum
TABLE II. LOS AND OVERALL COVERAGE PROBABILITY IN DIFFERENT SECTORISATION SCENARIOS.

\begin{tabular}{|l|c|c|}
\hline $\begin{array}{l}\text { Sectorisation } \\
\text { Order }\end{array}$ & $\begin{array}{l}\text { LOS Coverage } \\
\text { Probability (\%) }\end{array}$ & $\begin{array}{l}\text { Coverage } \\
\text { Probability (\%) }\end{array}$ \\
\hline 3 sectors & 51.00 & 55.67 \\
\hline 4 sectors & 63.50 & 64.17 \\
\hline 6 sectors & 67.33 & 67.33 \\
\hline 8 sectors & 75.67 & 92.83 \\
\hline $\mathbf{1 0}$ sectors & 75.17 & 78.17 \\
\hline
\end{tabular}

signal reception. As the steering angle (from the boresight) increase, the losses will increase. In three sectored site, the steering should cover at least $120^{\circ}$ $\left( \pm 60^{\circ}\right)$, however, with eight sectored site, steering requirements will decrease to only $45^{\circ}$, which will improve beamsteering efficiency as shown in fig.7.

The improvement comes in term of total interference, as UE's now can reject unwanted interference by steering their own patterns to the parent station. Rx Beamforming has improved received signal strength by steering its main lobe toward the direction of transmission.

TxBF has higher gain than $\mathrm{RxBF}$ due to the higher number of antennas that can be equipped in the base station. TxBF can simultaneously assign beams to user and improve cell-edge users due to high path gain and low interference. As shown in fig.8, TxBF outperform RxBF, which can accommodate higher pathloss and atmospheric attenuation, and improve SINR to fulfil quality of experience (QoE) provision in next generation $5 \mathrm{G}$ network.

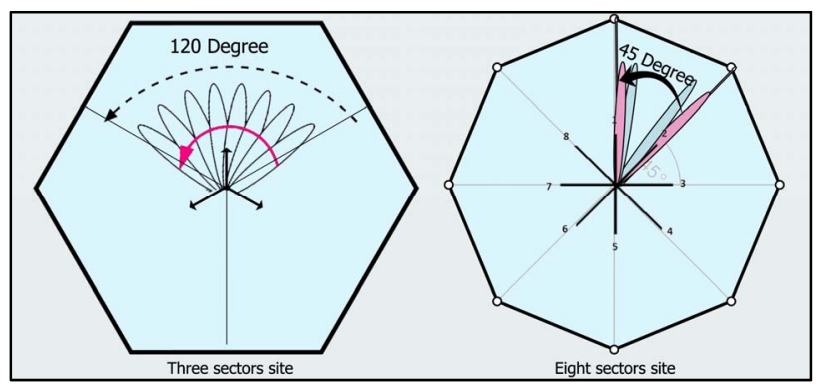

Fig.7, Sectorisation order effect on beamforming, left-side is 3 sectors and right-side is 8 sectors solution

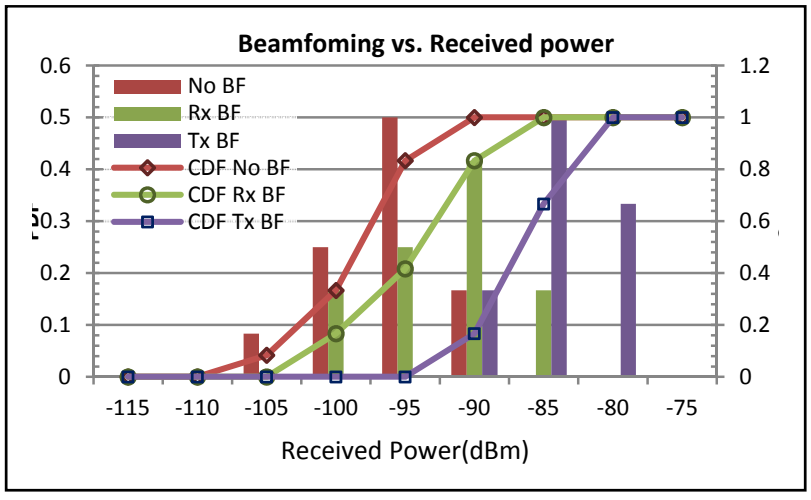

Fig. 8 , received power in $(\mathrm{dBm})$ with and without beamforming 
The data throughput of a user in these scenarios is highly impacted by the interference due to nearby sectors transmission. However, a very high data rates can be expected due to the densification in bandwidth, increase number of sectors, and beamforming.

As shown in fig.9, the data throughput has increased as the sectorisation increased (five smallcells base-stations are considered) with $2 \times 2$ multi input multi output MIMO antennas; however, this increment is impaired by high interference from nearby sectors. When beamforming is applied on ten sectors scenario, where the interference is very high, significant improvement is achieved in data rate, as shown in fig.9 (10 sectors with $\mathrm{BF}$ ), moreover, when interference among sectors is coordinated by inter cell interference coordination techniques in addition to beamforming, higher gain in data throughput can be achieved.

\section{CONCLUSION}

This paper introduces the higher order sectorisation scenario that can be applied in future $5 \mathrm{G}$ network operating in millimetre wave band, and evaluated system performance in term of data throughput and coverage probability through system level simulation. The simulation results show that high order sectorisation in millimetre wave band is capable of providing the necessary capacity increase and data speed for next generation network. In very low load factor, UE can experience a speed in excess of 1-4 Gbps. The higher order sectorisation is essential in busy urban areas to provide the necessary data rate, however, when the ISD is high, it is favourable to deploy new smallcell rather than sectorisation in order to improve coverage probability and increase the possibility of LOS transmission. Furthermore, beamforming is a powerful and key enabler technique to improve signal path and suppress interference. Therefore beamforming can highly improve data rate at user end and basestation end by increasing SINR.

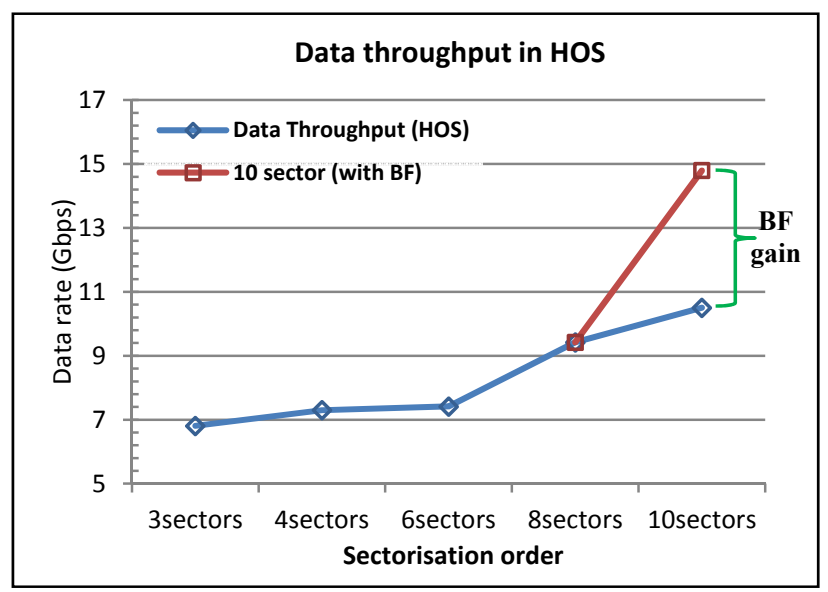

Fig.9, Base station data throughput in different sectorisation options and beamforming.

\section{REFERENCES}

[1] Qualcomm, "The 1000x Mobile Data Challenge," White paper. November, pp. 1-38, 2013.

[2] S. Chen and J. Zhao, "The requirements, challenges, and technologies for $5 \mathrm{G}$ of terrestrial mobile telecommunication," IEEE Commun. Mag., vol. 52, no. 5, pp. 36-43, May 2014.

[3] Ericsson, "5G : what is it ?," White Pap., pp. 1-10, 2014.

[4] N. Al-Falahy and O. Alani, "Potential Technologies to 5G Network: Challenges and Opportunities," IEEE IT Profesional, 2016, in press.

[5] A. Wacker, J. Laiho-Steffens, K. Sipila, and K. Heiska, "The impact of the base station sectorisation on WCDMA radio network performance," in IEEE 50th Vehicular Technology Conference Gateway to 21st Century Communications Village, 1999, pp. 1-5.

[6] J. Laiho-Steffens, A. Wacker, and P. Aikio, "The impact of the radio network planning and site configuration on the WCDMA network capacity and quality of service," in IEEE 51st Vehicular Technology Conference Proceedings VTC2000-Spring, 2000, pp. 1006-1010.

[7] O. Yilmaz, S. Hamalainen, and J. Hamalainen, "System level analysis of vertical sectorization for 3GPP LTE," in 6th International Symposium on Wireless Communication Systems, 2009, pp. 453-457.

[8] J. Acharya and S. Akoum, "Optimizing Vertical Sectorization for High-Rises," in International Workshop on Emerging MIMO Technologies with 2D Antenna Array for 4G LTE-Advanced and 5G, ICNC Workshop, 2015, pp. 1-5.

[9] X. Ge, H. Cheng, M. Guizani, and T. Han, "5G wireless backhaul networks: challenges and research advances," IEEE Netw., vol. 28, no. 6, pp. 6-11, 2014.

[10] F. Athley, M. N. Johansson, and A. Nilsson, "Increased Sectorization: Horizontal or Vertical ?," in IEEE 78th Vehicular Technology Conference (VTC Fall), 2013, pp. 1-5.

[11] S. Akoum, O. El Ayach, and R. W. Heath, "Coverage and capacity in mmWave cellular systems," in (ASILOMAR) Conference on Signals, Systems and Computers, 2012, pp. 688-692.

[12] T. Kim, I. Bang, and D. K. Sung, "Design Criteria on a mmWavebased Small Cell with Directional Antennas," in IEEE 25th International Symposium on Personal, Indoor and Mobile Radio Communications, 2014, pp. 103-107.

[13] S. Rajagopal, S. Abu-Surra, and F. Khan, "Antenna Array Design for Multi-Gbps mmWave Mobile Broadband Communication," in IEEE Global Telecommunications Conference - GLOBECOM, 2011, pp. 1-6.

[14] Remcom Inc., "XFdtd Reference Manual." [Online]. Available: http://www.remcom.com/xf7. [Accessed: 15-Jan-2016].

[15] S. G. Larew, T. A. Thomas, M. Cudak, and A. Ghosh, "Air Interface Design and Ray Tracing Study for 5G Millimeter Wave Communications," in IEEE Globecom Workshops, 2013, pp. 117122.

[16] Remcom Inc., “Wireless InSite.," 2014. [Online]. Available: www.remcom.com/wireless-insite. [Accessed: 15-Jan-2016].

[17] M. R. Akdeniz, et al, "Millimeter Wave Channel Modeling and Cellular Capacity Evaluation," IEEE J. Sel. AREAS Commun., vol. 32, no. 6, pp. 1164-1179, 2014.

[18] M. N. Kulkarni, T. A. Thomas, F. W. Vook, A. Ghosh, and E. Visotsky, "Coverage and Rate Trends in Moderate and High Bandwidth 5G Networks," in Globecom Workshop, 2014, pp. 422426.

[19] N. Bhushan, et al, "Network Densification: The Dominant Theme for Wireless Evolution into 5G," IEEE Commun. Mag., vol. 52, no. 2, pp. 82-89, 2014. 